\title{
El control glucémico estricto en pacientes con diabetes tipo 2 disminuye los eventos cardiovasailares pero no la mortalidad en un seguimiento por diez años
}

Tigh glycemic control in patients with type 2 diabetes reduces cardiovascular events but not mortality in a follow up for ten years

\section{Objetivos}

Evaluar eventos cardiovasculares mayores en pacientes con diabetes 2 que habían participado del ensayo VADT (acrónimo del inglés Veterans Affairs Diabetes Trial) a los 9,8 años en promedio de iniciado el estudio. El mismo había mostrado previamente que el control glucémico intensivo comparado con el tratamiento estándar no reduce significativamente la tasa de eventos cardiovasculares mayores entre 1.791 veteranos de guerra con diabetes tipo 2 durante un seguimiento medio de 5,6 años.

\section{Diseño, lugar y pacientes}

Cohorte prospectiva observacional. Realizado en diez centros de Estados Unidos (EE.UU). Una vez finalizado el ensayo VADT, los pacientes volvieron a su terapéutica habitual y se realizó un seguimiento de 1.655 de ellos (92,4\% de la cohorte original) usando bases de datos para identificar procedimientos, hospitaliza-
Hayward R y col. N Engl J Med 2015;372:2197-206. ciones y muerte. La mayoría de los participantes $(77,7 \%)$ completaron además una encuesta anual para detectar eventos que no fueran fácilmente reconocidos en la base de datos.

\section{Medición de resultados principales}

El resultado principal fue el tiempo para el primer evento cardiovascular mayor (ataque cardiaco, accidente cerebro vascular, desarrollo o empeoramiento de insuficiencia cardíaca, amputación por gangrena isquémica o muerte de causa cardiovascular). Los resultados secundarios fueron mortalidad cardiovascular y mortalidad por todas las causas. El análisis se realizó por intención de tratar.

\section{Resultados principales y secundarios}

El resultado principal fue analizado de la cohorte que realizó la encuesta, a diferencia de los resultados secundarios que fueron analizados de la cohorte total de seguimiento (Tabla 1).

Tabla 1. Resultados principales al final del seguimiento.

\begin{tabular}{|c|c|c|c|c|c|}
\hline \multirow{2}{*}{ Desenlace } & \multicolumn{2}{|c|}{ Terapia estándar } & \multicolumn{2}{|c|}{ Terapia intensiva } & \multirow{2}{*}{$\begin{array}{l}\text { Hazard Ratio } \\
\text { (95\% IC) }\end{array}$} \\
\hline & $\begin{array}{c}\text { Eventos } \\
\text { (n participantes/n total) }\end{array}$ & $\begin{array}{c}\text { Tasa de } \\
\text { incidencia** }\end{array}$ & $\begin{array}{c}\text { Eventos } \\
\text { (n participantes/n total) }\end{array}$ & $\begin{array}{c}\text { Tasa de } \\
\text { incidencia* }\end{array}$ & \\
\hline \multicolumn{6}{|l|}{ Primario } \\
\hline Evento cardiovascular mayor & 288/688 & 52,7 & $253 / 703$ & 44,1 & $0.83(0,70$ a 0,99$)$ \\
\hline \multicolumn{6}{|l|}{ Secundarios } \\
\hline Muerte de causa cardiovascular & $83 / 818$ & 11,3 & $74 / 837$ & 10,0 & $0,88(0,64$ a 1,20$)$ \\
\hline Muerte de cualquier causa & $258 / 818$ & 30,3 & $275 / 837$ & 32,0 & $1,05(0,89$ a 1,25$)$ \\
\hline
\end{tabular}

*Número de eventos por 1.000 personas/año de seguimiento. 95\% IC: intervalo de confianza del 95\%

\section{Conclusión}

Después de casi diez años de seguimiento, los pacientes con diabetes tipo 2 que habían sido asignados en forma aleatoria al grupo de control glucémico intensivo por 5,6 años, tuvieron 8,6 menos eventos cardiovasculares mayores por cada 1.000 personas-año que aquellos asignados al grupo de terapéutica estándar, pero no se observó aumento en la tasa de supervivencia total.

Fuente de financiamiento: National Institute of Diabetes and Digestive and Kidney Diseases y National Institutes of Health, de EE.UU. Conflictos de interés: Dr. Reaven (AstraZeneca, NovoNordisk y Amgen) y Dr. Emanuele (Merk).

\section{Comentario}

Se sabe que la hiperglucemia se asocia a enfermedad cardiovascular (ECV) y que el control glucémico estricto disminuye las complicaciones microvasculares (retinopatía, nefropatía, etc.) en los pacientes diabéticos tipo 2. Menos claro es si disminuye el riesgo de enfermedad macrovascular, principal causa de muerte de estos pacientes. Sobre esta última cuestión se han publicado diferentes ensayos clínicos con resultados variables, debidos probablemente a que se trataban de diferentes poblaciones y objetivos glucémicos. El estudio VADT incluyó personas con diabetes de larga evolución (promedio 11,5 años), añosos (edad promedio 60 años) y una significativa proporción de antecedentes de ECV previa (40\%). Aunque al final del estudio (5,6 años) la rama intensiva llegó a una hemoglobina glicosilada (Hba1c) media de $6,9 \%$, no se observaron diferencias en el riesgo de desarrollar ECV ${ }^{1}$. Por el contrario, en el seguimiento de casi 10 años tuvieron "apenas" menor incidencia de eventos pero no mayor ni menor mortalidad cardiovascular (CV). Este resultado difiere del documentado por el estudio UKPDS (acrónimo del inglés United Kindom Prospective Diabetes Study), en el cual, luego de diez años de tratamiento intensivo (Hba1c menor 7,4\%) en pacientes con diabetes de reciente diagnóstico, más jóvenes (promedio de edad, 53 años) y sin ECV previa se observó disminución tanto de la tasa de eventos como de la mortalidad $\mathrm{CV}^{2}$. En el otro extremo, el estudio ACCORD (acrónimo del inglés Action to Control Cardiovascular Risk in Diabetes), con pacientes muy similares al VADT pero un objetivo de Hba1c menor a $6 \%$, tuvo que suspenderse a los 3,5 años por documentarse mayor mortalidad CV en la rama intensiva, con una alta incidencia de hipoglucemias ${ }^{3}$. Finalmente, en el estudio ADVANCE (acrónimo del inglés Action in Diabetes and Vascular Disease: Preterax and Diamicron Modified Release Controlled Evaluation) el control glucémico tuvo un efecto neutro ${ }^{4}$.

\section{Conclusión del comentador}

Salvo en pacientes diabéticos 2 de reciente diagnóstico, no añosos y sin ECV donde el control glucémico estricto disminuiría el riesgo de eventos y muertes cardiovasculares, el tratamiento hipoglucemiante debería individualizarse de acuerdo a la preferencia del paciente y su riesgo de hipoglucemia.

\section{Albano Dalibón [ Servicio de Endocrinología y Metabolismo del Hospital Italiano de Buenos Aires. albano.dalibon@hiba.org.ar ]}

Dalibón A. El control glucémico estricto en pacientes con diabetes tipo 2 disminuye los eventos cardiovasculares pero no la mortalidad en un seguimiento por diez años. Evid Act Pract Ambul. $2016 ; 19(3) 85$. Comentado de: Hayward RA, y col. Follow-up of glycemic control and cardiovascular outcomes in type 2 diabetes. NEJM. 2015;372(23):2197-206. PMID: 26039600

Referencias:

1. Duckworth W, y col. Glucose Control and Vascular Complications in Veterans with Type 2 Diabetes. N Engl J Med. 2009;360(2):129-39.

2. Tumer R. Effect of intensive blood-glucose control with metformin on complications in overweight patients with type 2 diabetes (UKPDS 34). Lancet. 1998;352(9131):854-65.

3. The Action to Control Cardiovascular Risk in Diabetes Study Group. Effects of Intensive Glucose Lowering in Typo 2 Diabetes. N Engl J Med. 2008;358(24):2545-59.

4. The ADVANCE Collaborative Group. Intensive Blood Glucose Control and Vascular Outcomes in Patients with Type 2 Diabetes. N Engl J Med. 2008;358(24):2560-72. 